This article has been scanned by iThenticat No plagiarism detected

Volume 3, Issue 4, August 2021

p. $129-137$

\title{
CHALLENGES OF PALESTINIAN WOMEN'S PARTICIPATION IN HIGHER LEADERSHIP POSITIONS IN EDUCATIONAL INSTITUTIONS AND WAY TO OVERCOME THEM
}

http://dx.doi.org/10.47832/2757-5403.4-3.12

\section{Rawan Wael SIAJ1}

\begin{abstract}
:
The purpose of this study to identify the challenges facing the participation of Palestinian women in assuming higher educational leadership positions in the various Palestinian educational institutions and higher education institutions. The study shed light on possible ways to overcome these challenges. The sample of the study consisted of twelve Palestinian women working in different institutions with different job titles, and they were chosen in an intentional and accessible way. To achieve the objectives of the study, a qualitative approach was used to answer the study questions. The semi-closed interview tool was used, and the responses of the respondents were organized into patterns and answers.

The results of the study showed a great similarity between the challenges that Palestinian women face locally and the challenges that face women in different countries globally. Such as the lack of awareness of women themselves about their rights, unprofessional jealousy among women themselves at work, lack of societal support for women and underestimating the importance of their roles, the stereotype of women resulting from a defect in the value system and upbringing among members of society, the spread of violence towards women in conservative cities, and other defects in administrative organization within some educational institutions. But what distinguished the reality of the challenges was the current political situation under the Israeli occupation, which constituted an additional obstacle. Such as the difficulty of moving and traveling freely is the long hours that women spend in the racist Israeli security check stations that are scattered between cities and governorates.

In light of the results of the study, the researcher concluded a set of recommendations, the most important of which are: Work to empower Palestinian women in their work by holding courses and workshops that
\end{abstract}

\footnotetext{
${ }^{1}$ Dr. , The Ministry of Education, Palestine, rawansiajubeh@gmail.com, https://orcid.org/0000.0002-7722-5932
}

Copyright $(\mathcal{C}$ Published by IJHER Journal, www.ijherjournal.com

Rimar Academy, Fatih, Istanbul, 34093 Turkey

All rights reserved 


\section{CHALLENGES OF PALESTINIAN WOMEN'S PARTICIPATION IN HIGHER \\ LEADERSHIP POSITIONS IN EDUCATIONAL INSTITUTIONS AND WAY TO \\ OVERCOME THEM}

seek to increase Palestinian women's knowledge of their rights, raise their confidence in themselves and their work, and hold community courses within various higher education institutions that will clarify the importance of the role of women in society and work to change the negative stereotype they have towards women, And work on issuing regulations and legislation that would allocate free hours for women to be able to write and produce scientifically.

Challenges of Palestinian Women's Participation, Educational Leadership Centers.

Key words: Eeducational Administration Position, The Challenges Facing Women.

\section{تحديات مشاركة المرأة القلسطينية في المراكز القيادية العليا في المؤسسات التربوية

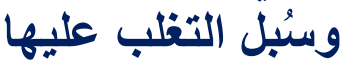

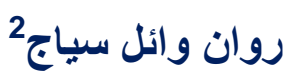

\begin{tabular}{|c|}
\hline 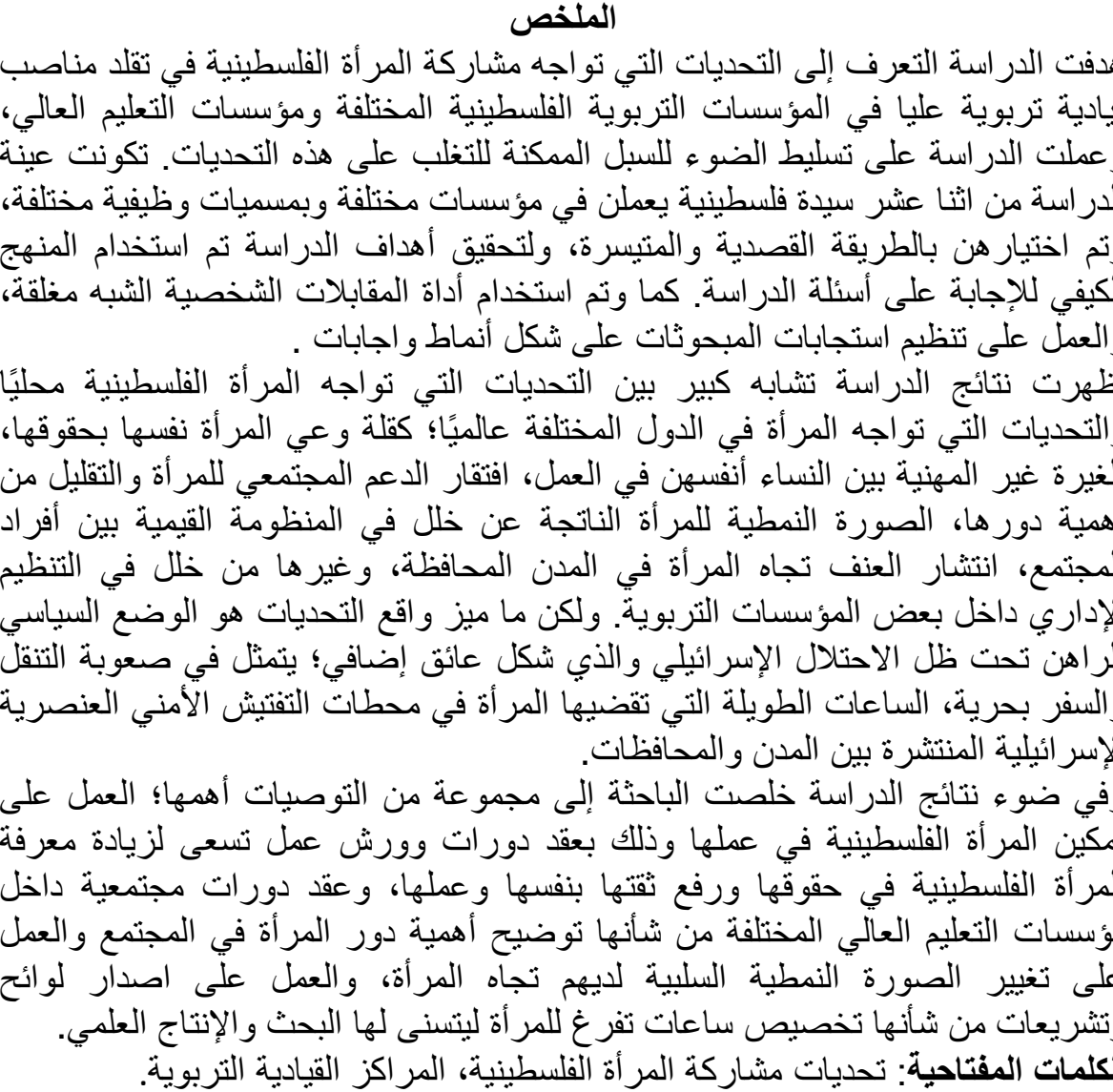 \\
\hline
\end{tabular}

2² د. ، وزارة التربية، فلسطين، rawansiajubeh@gmail.com 
تلعب المر أة دوراً كبير اً بل أساسياً في المجتمعات؛ مما لا شك فيه أنها نصف المجتمع، وهي عنصر فعَال في التنمية

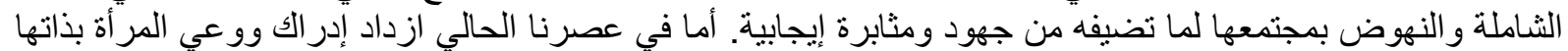

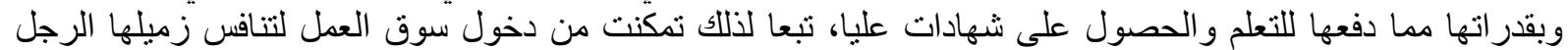

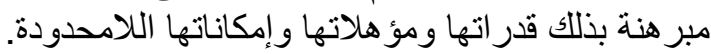

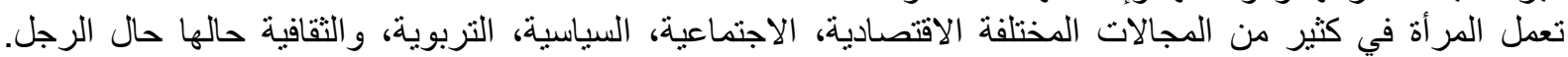

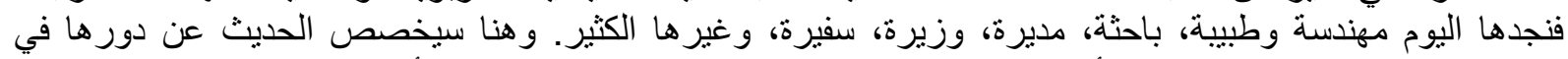

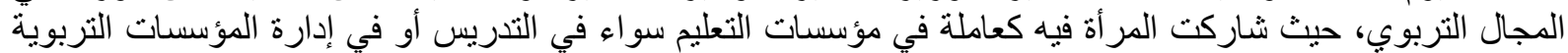

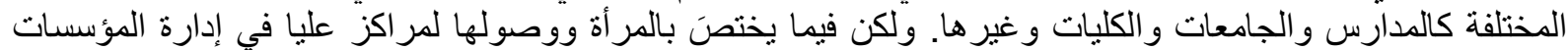

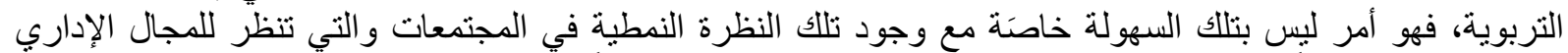

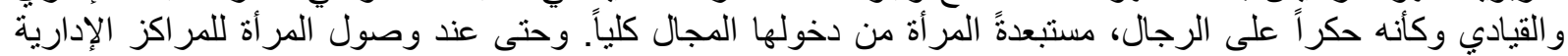
فإنه يتم تعيينها في الإدارات الوسطى أو الثانوية وليس في الإدارات الئات العليال(Lemasters \& Roach, 2016).

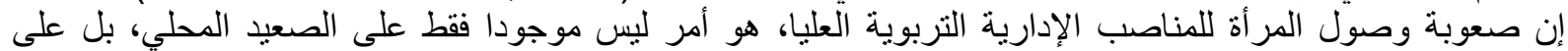

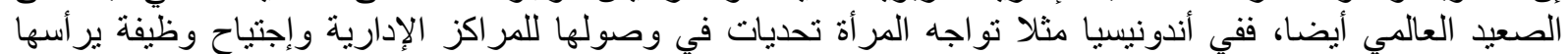

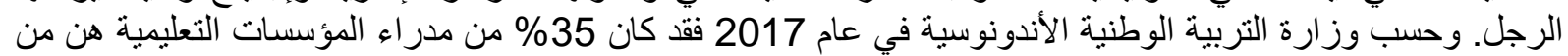

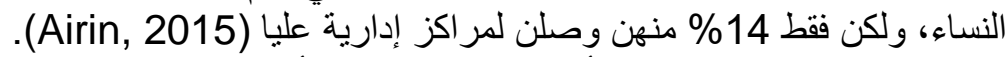

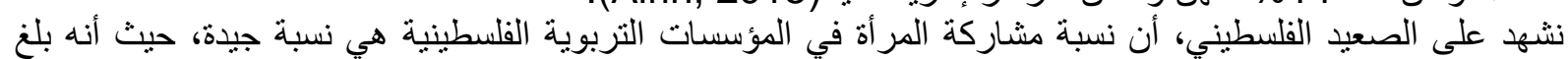

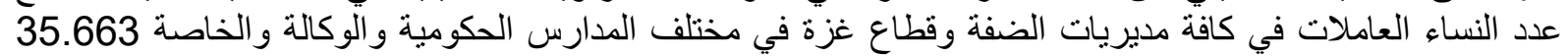

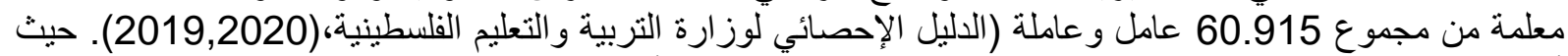

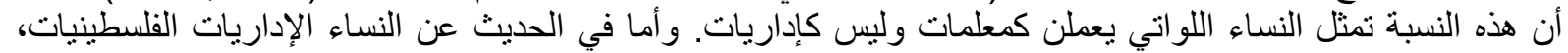

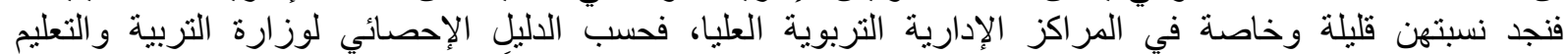

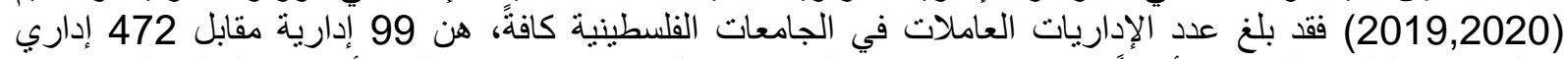

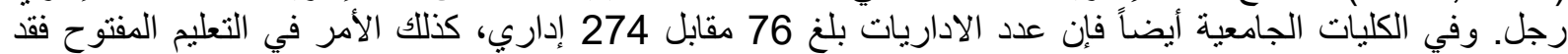
وصل عدد الإداريات الى 354 مقابل 1583 إداري.

\section{الأدب المفاهيمي وتثكل المفاهيم الآتية الإطار المفاهيمي الذي اعتمدته هذه الدراسة.

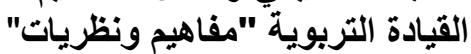

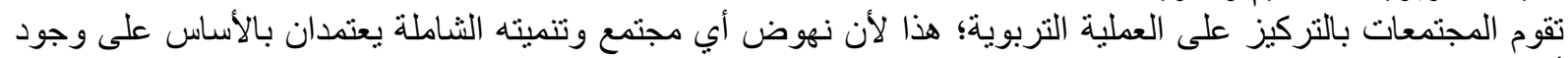

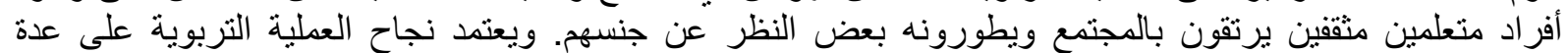

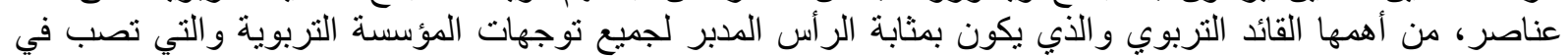

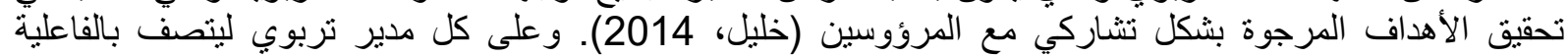

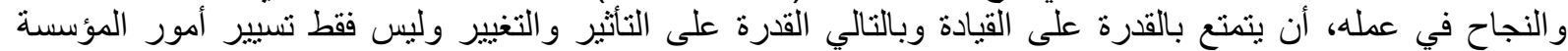

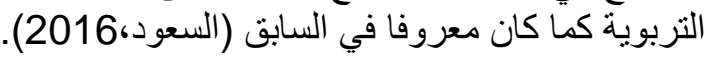

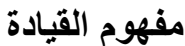

\section{عرفها السعود (2016) على ألى أنها:}

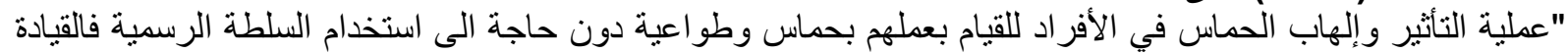

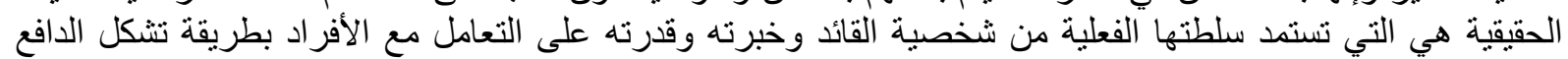

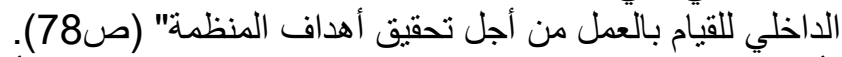

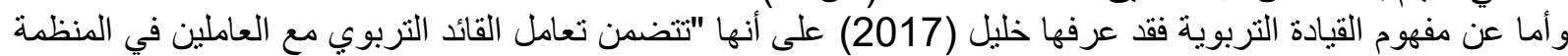

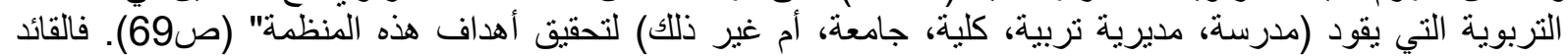

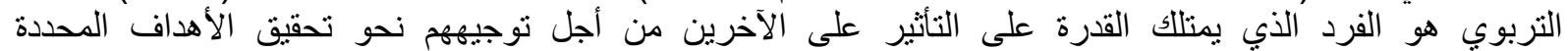

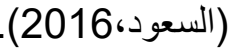

عناصر القيادة التربوية

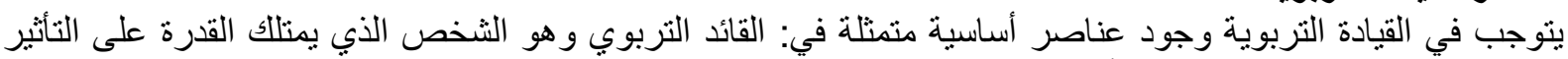

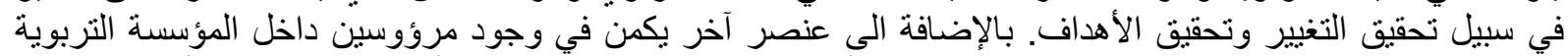

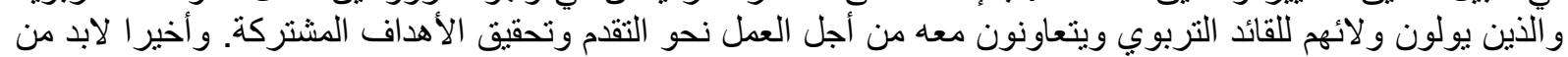
وجود الأهداف التربوية و التي تتمنل في الغايات التي تسعى المؤسسة التربوية لتحقيقها (خليل، 2017). 


\section{CHALLENGES OF PALESTINIAN WOMEN'S PARTICIPATION IN HIGHER \\ LEADERSHIP POSITIONS IN EDUCATIONAL INSTITUTIONS AND WAY TO \\ OVERCOME THEM}

وظائف القائد التربوي

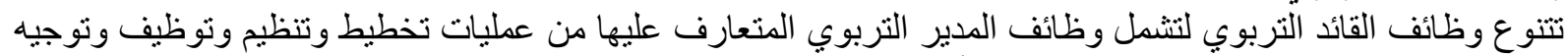

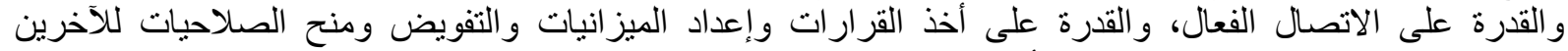

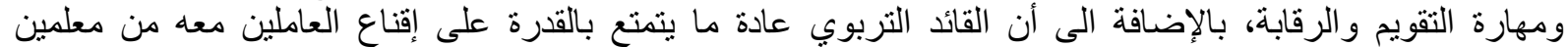

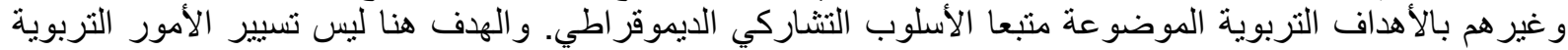

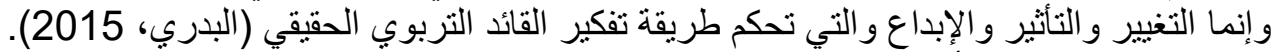

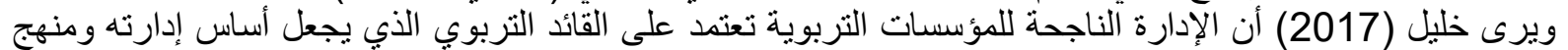

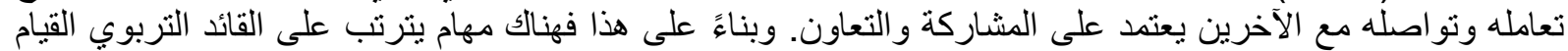

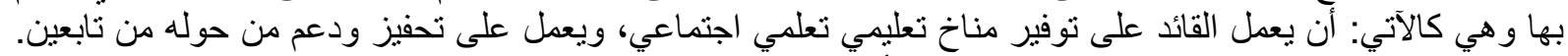

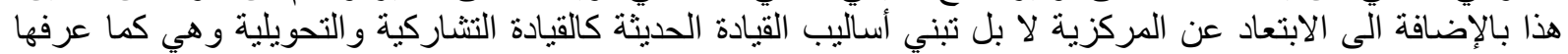

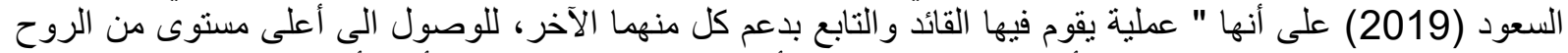

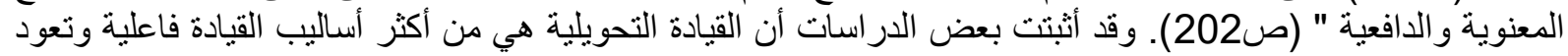

بالنجاح على القائد و المؤسسة التربوية على على حد سواء (Chin,2011).

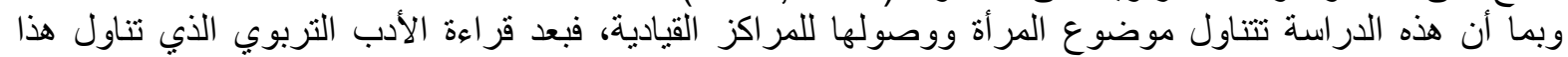

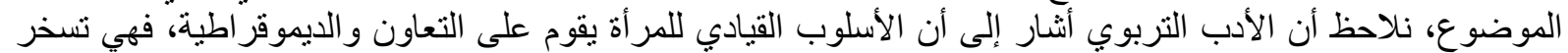

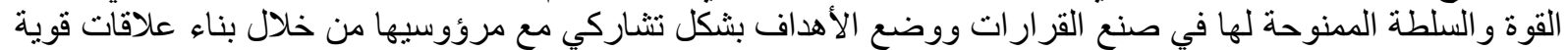

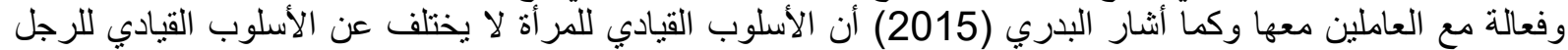

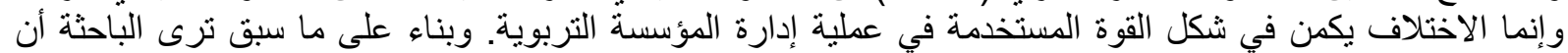

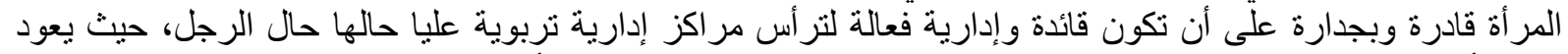

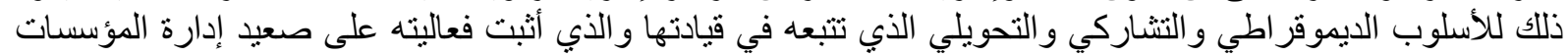
التربوية.

أتضح بعد مر اجعة الأدب التربوي، أنه نم تقسيم المعيقات التي تواجه المر أة في الوصول للمر اكز الإدارية التربوية العليا

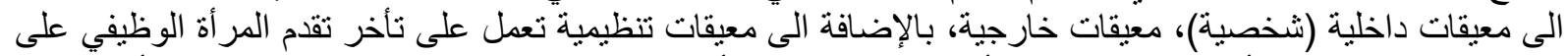

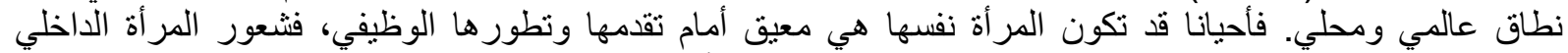

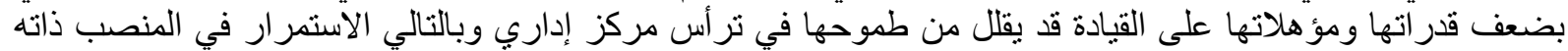

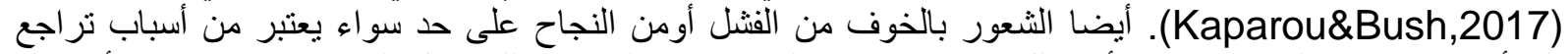

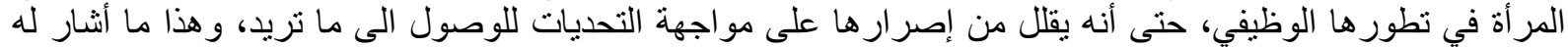

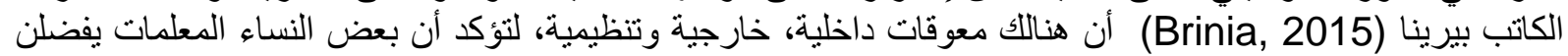

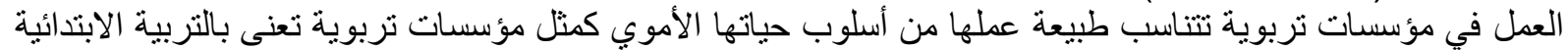

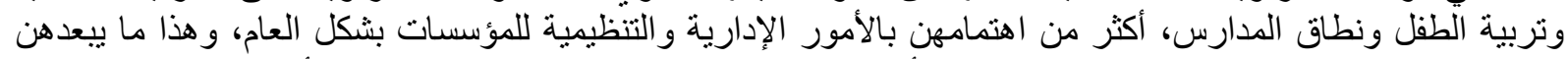

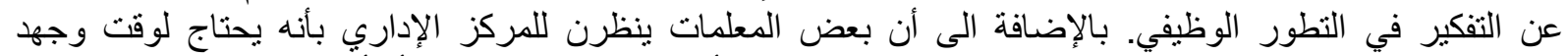

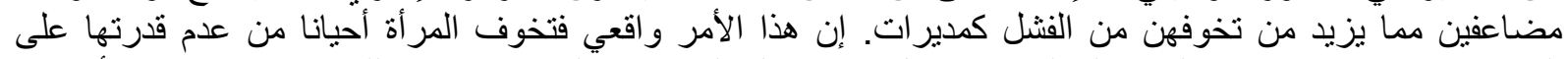

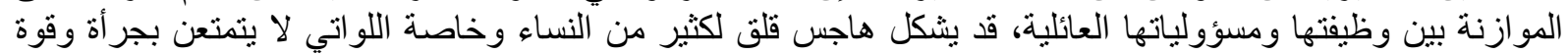

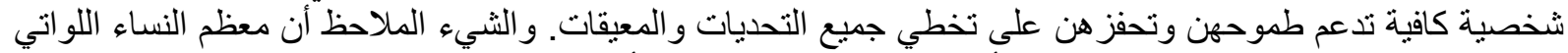

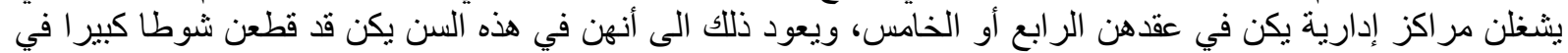

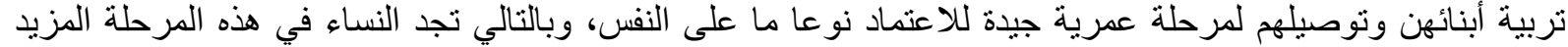
من الوقت للتفرغ لمنطلبات المركز الإداري (Lemasters \& Roach, 2016). مشكلة الاراسة وأهدافهها

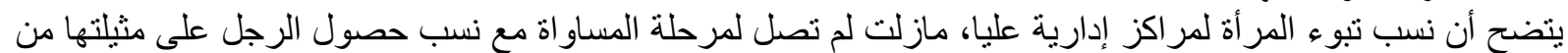

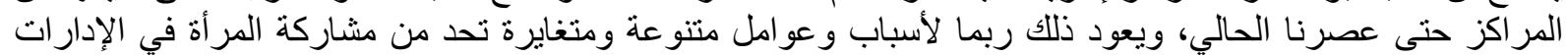

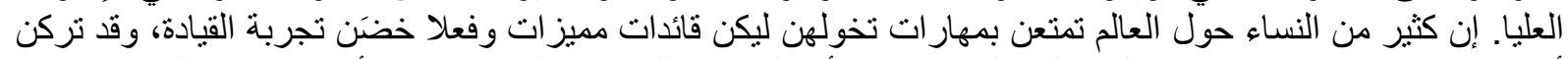

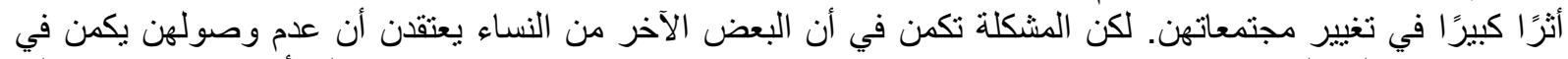

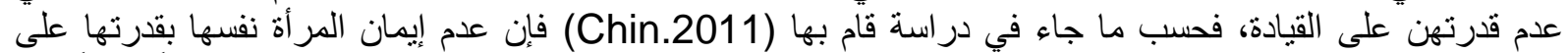

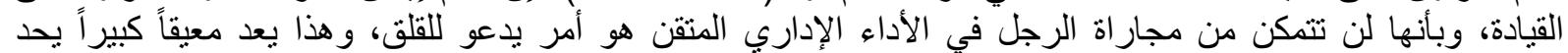

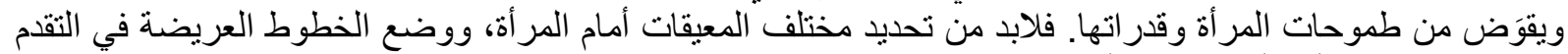

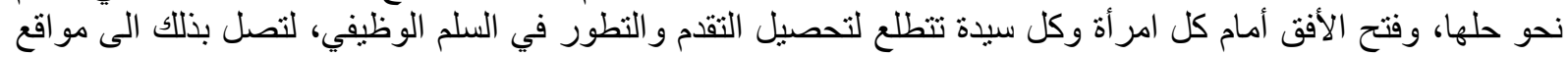


إدارية عليا كعميدة كلية في إحدى الجامعات الفلسطينية أو حتى تتقلد منصب رئاسة جامعة أو حتى منصب وزير القيرة التربية

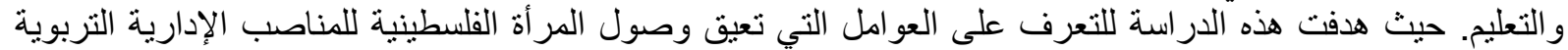

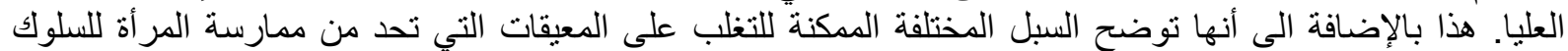
القيادي في المر اكز الإدارية العليا بمحافظة العاف رام الله و البيرة.

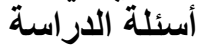

تبعا لما سبث تسعى هذه الدراسة اسلة للإجابة عن الأسئلة الآتية:

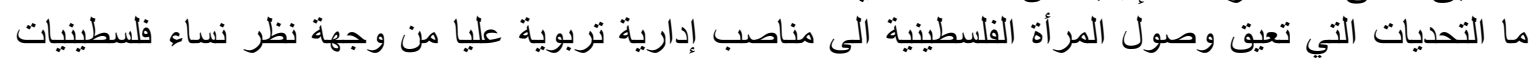

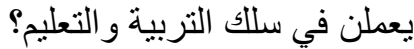
ما سبل التغلب على المعيقات التي تحول دون ممارسة المرأة للسلوك القيادي ووصولها للمر اكز الإدارية العليا من

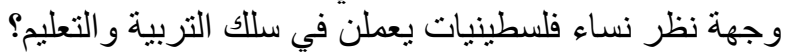

أهمية الاراسة أنة

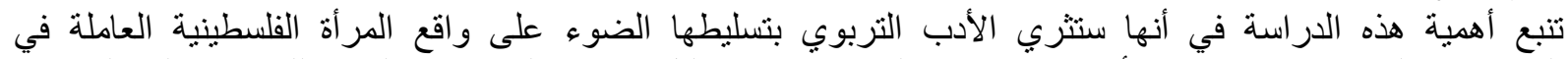

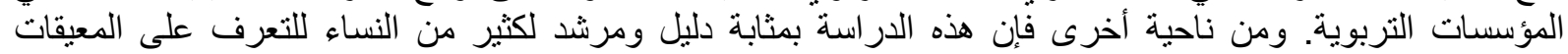

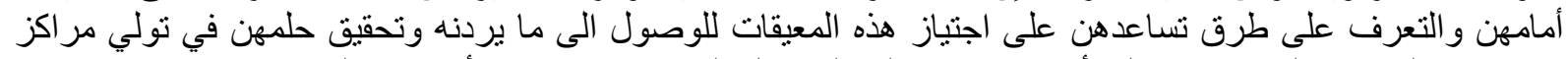

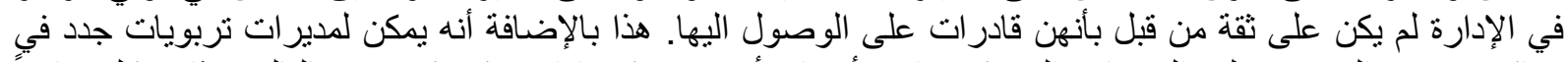

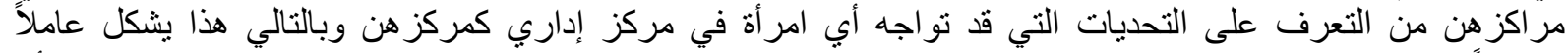

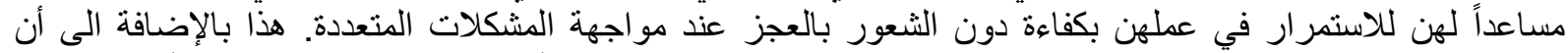

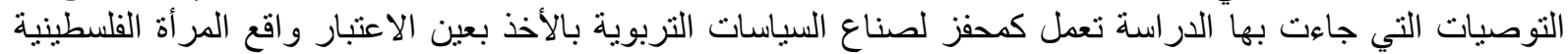

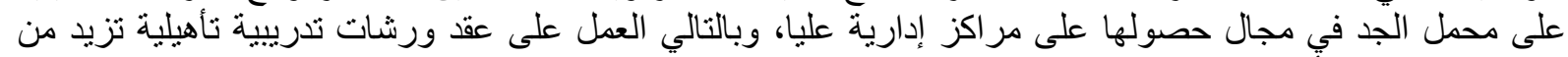
كفاءة وقدرات المر أَة القيادية وتعمل على دعمها في الوصول للمر اكز الإدارية التربوية حالها حال الرجل الرجل الفلسطيني في تلالي

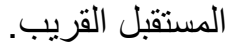
مصطلحات الاراسة

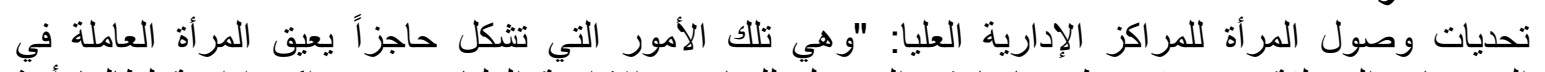

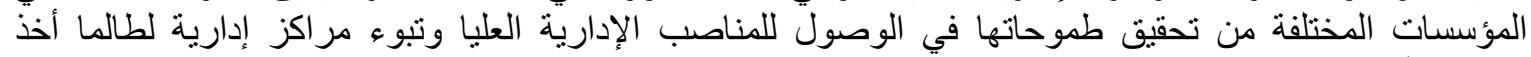

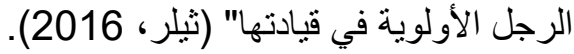

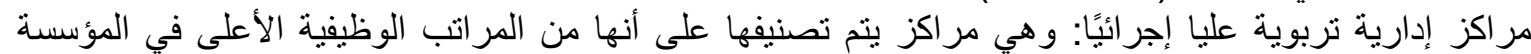

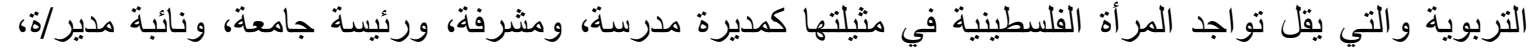

و عميدة كلية ووزيرة للتربية و التعليم و غير ها. حلدود الارسة اسنة تتمثل حدود الدر اسة في الآتي: الحد الموضو عي: تمثلت بدر اسة تحديات مشاركة المر أة الفلسطينية في المر اكز القيادية العليا في المؤسسات التربوية

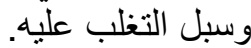
الحد المكاني: طبقت هذه الدمانبة الدة في القدس ومحافظة رام الله والبيرة.

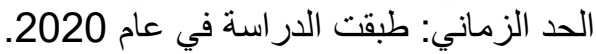

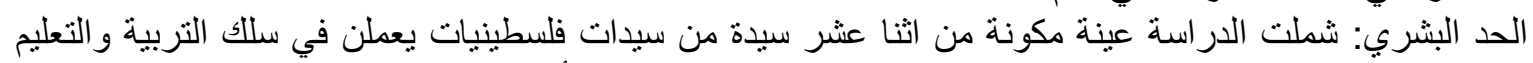

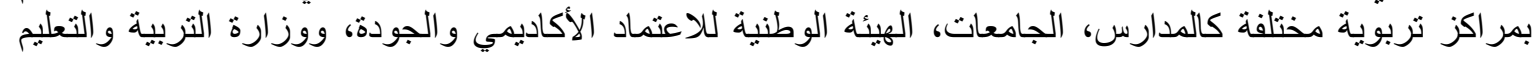

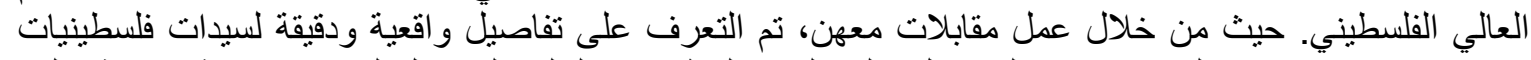

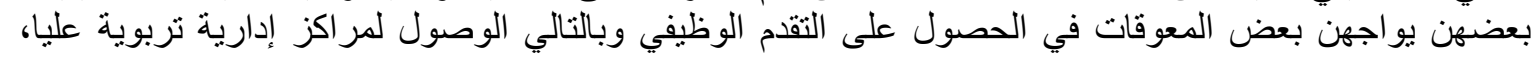
وبعضهن الآخر يواجهن بعض التحديات كونهن يعملن في مر اكز إدارية تربوية علية العيا في مؤسسات تربوية فلسطينية

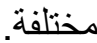

الار اسات السابقة

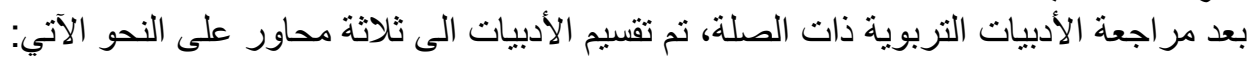

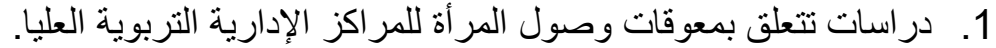
2.

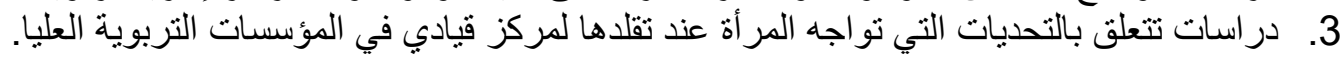

المحور الأول: دراسات تتعلق بمعوقات وصول المرأة للمراكز الإدارية التربوية العليا.

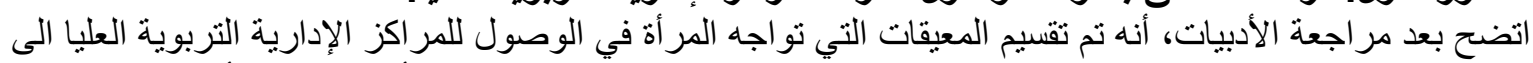

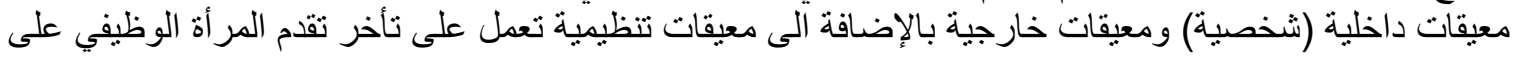




\section{CHALLENGES OF PALESTINIAN WOMEN'S PARTICIPATION IN HIGHER \\ LEADERSHIP POSITIONS IN EDUCATIONAL INSTITUTIONS AND WAY TO \\ OVERCOME THEM}

نطاق عالمي ومحلي. فأحيانا قد تكون المر أة نفسها هي معيق أمام تقدمها وتطور ها الوظيفي، فتشعور المر أة الداخلي

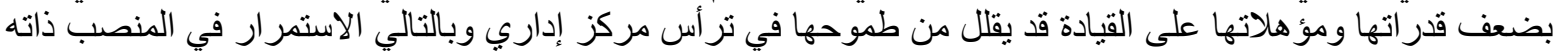

(Kaparou\&Bush,2017)

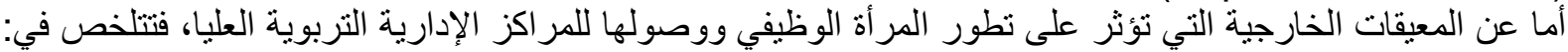

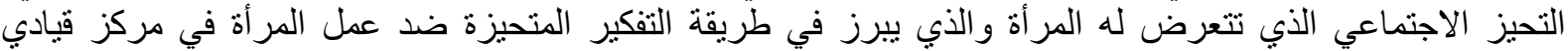
و إداري عالي، و هذه ظاهرة توجد في غالبية المجتمعات و الثقافات حول العالم Kaparou \& Bush, 2017; Liljia

.\& Luddeckens, 2016)

المحور الثاني: دراسات توضح التناقض الموجود حول قدرة المرأة على القيادة والوصول لمراكز إدارية تربوية عليا.

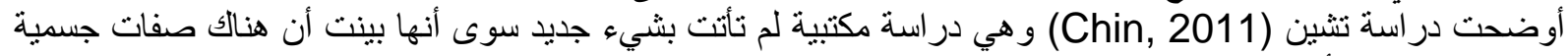

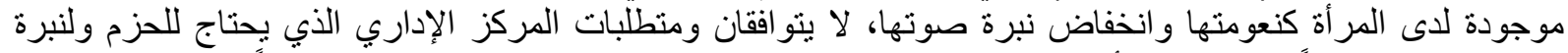

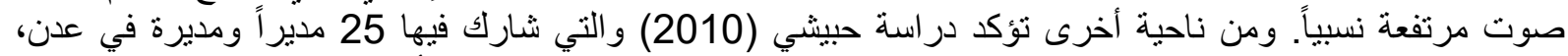

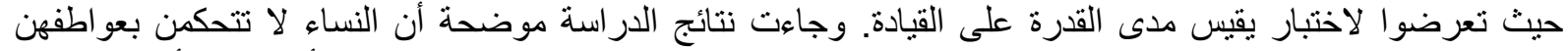

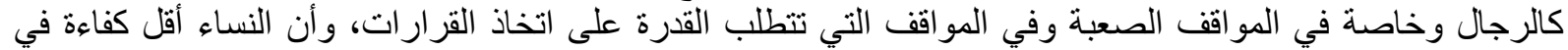

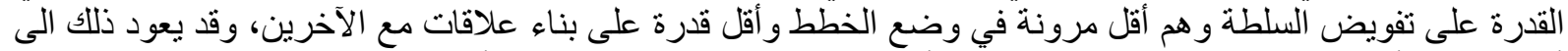

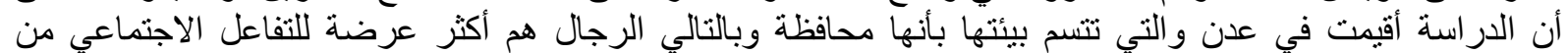

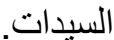

المحور الثالث: دراسات تتعلق بالتحديات التي تواجه المرأة عند تقلدها لمركز في الإدارة التربوية العليا.

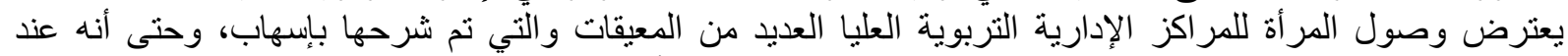

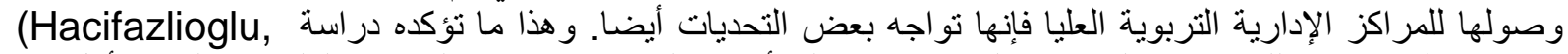

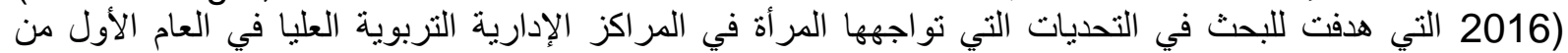

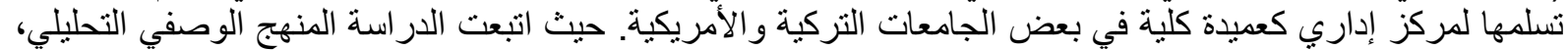

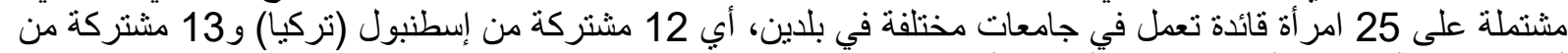

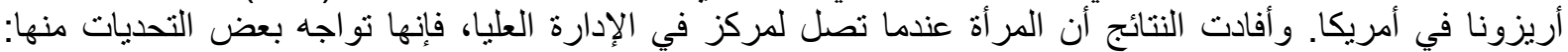

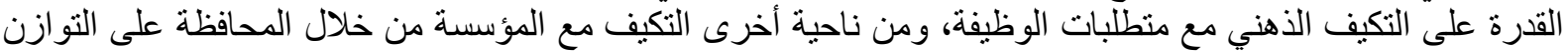

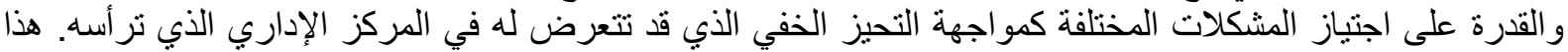

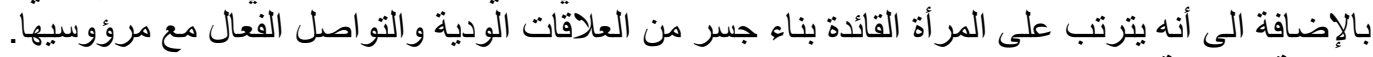

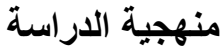

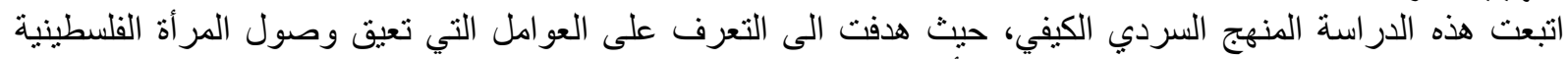

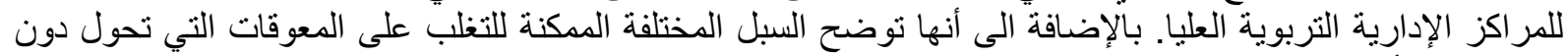
ممارسة المر أة للسلوك القيادي للوبة اللوصول اللى المر اكز الإدارية التربوية العليا بمحافظة رام الله والبيرة. مجتمع الاراسة المنة تكون مجتمع الدراسة من النساء الفلسطينيات اللوات بعملن في المؤسسات التربوية الثناث، أولها وزارة التربية التربية والتعليم الفلسطينية، جامعة بيرزيت، بالإضافة هيئة الاعتماد الأكاديمي و الجودة (AQAC).

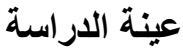

تم اختيار عينة الدراسة من المؤسسات التربوية بشكل قصدي وعينة متيسرة لتشكل اثتتا عشر سيدة تربوية فلسطينية يعملن في سلك التربية و التعليم العالي بمسميات وظيفية التئية مختلفة. أدوات الأراسة في التانة

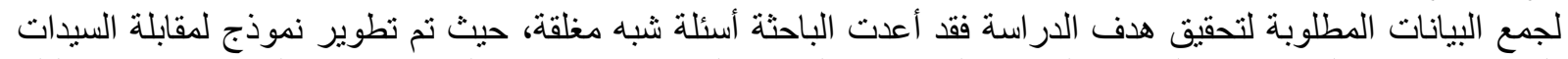

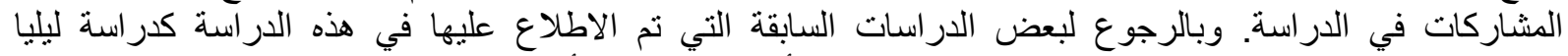
ولوديكينز (Lilja \& Luddeckens, 2016)، وذللك من أجل الإجابة عن أسئلة الدراسة. صدق الأداة وثباتها

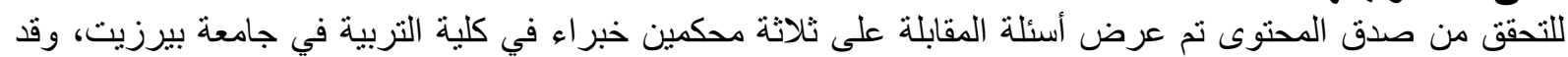

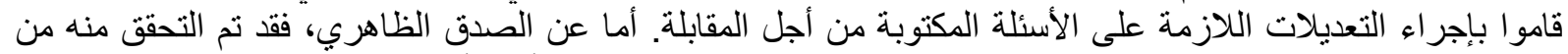
خلال قيام الباحثة بعمل مقابلة مع سيدتين كعينة استطلاعية، وذلك للتحقق من أن الأسئلة المطروحة كانت فعالة وتخدم 


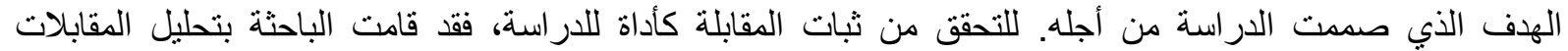

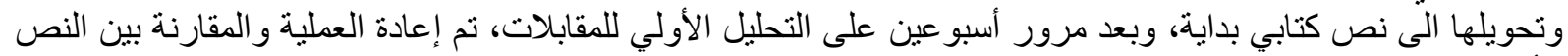

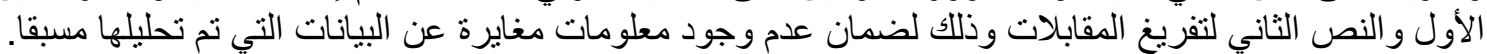

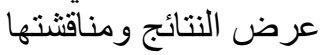

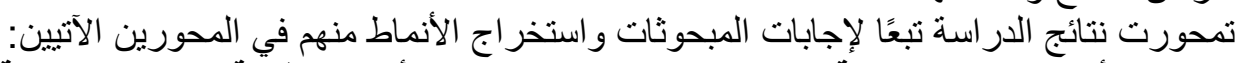

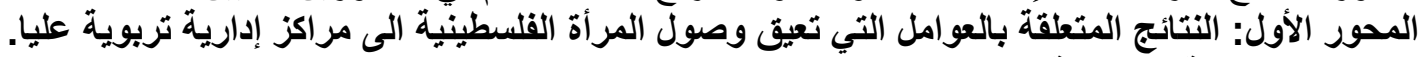

1 1 الغيقة داخلية (شخصية):

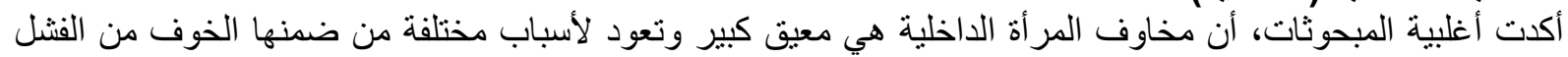

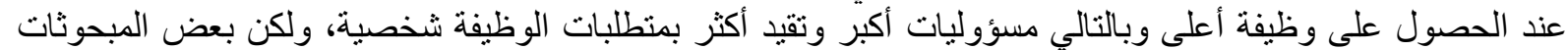

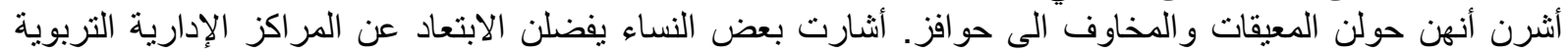

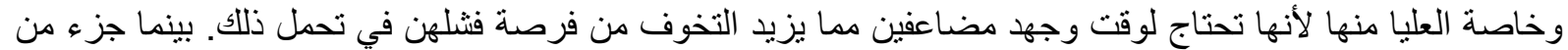

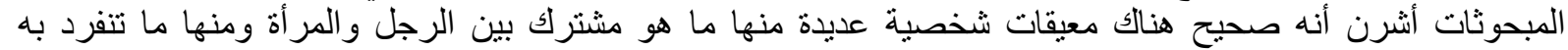

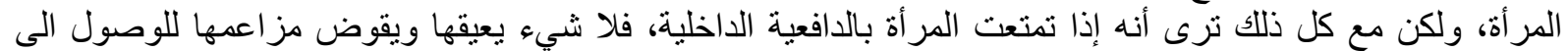
أعلى المر اكز الإدارية التربوية.

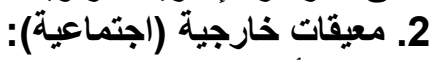

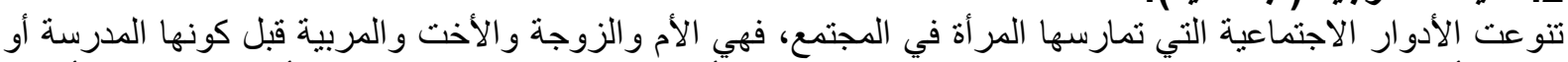

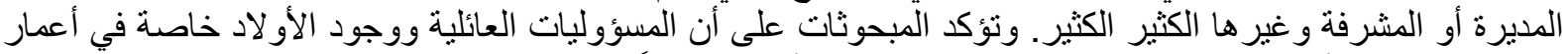

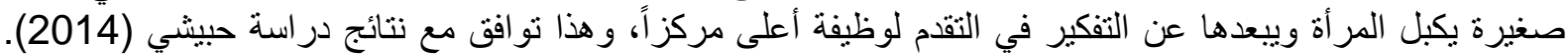

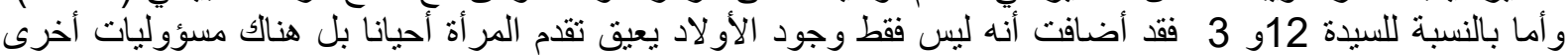

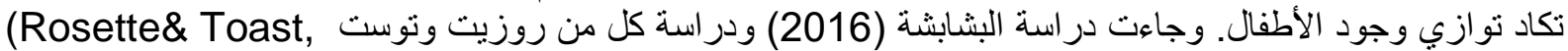

ومن ناحية أخرى تندرج نظرة المجتمع المتحيزة تحت مسمى المعيقات الاجتماعية مشكلة معيق آخر ، وذللك لوجود التأثير

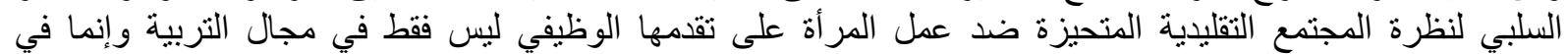

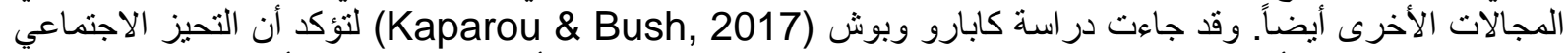

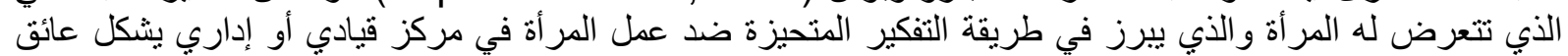

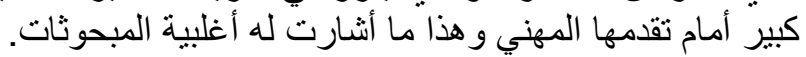

3. معيقات تنظيمية (وظيفية):

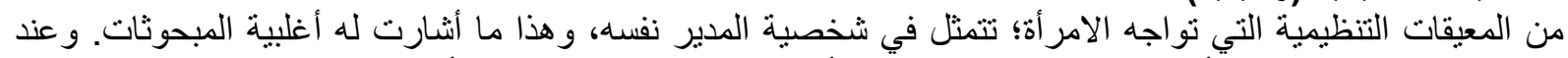

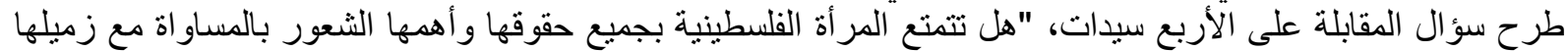

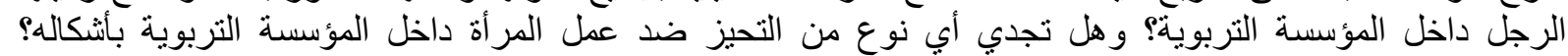

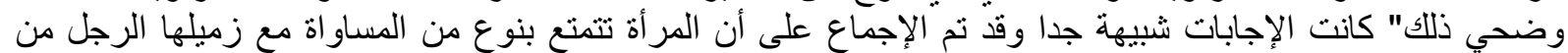

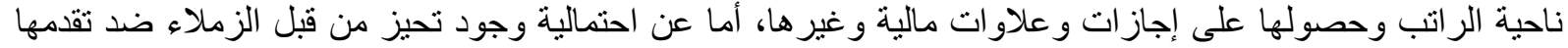

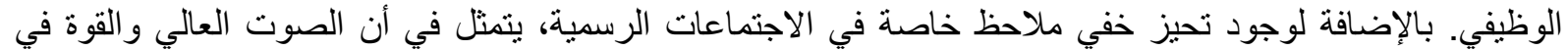

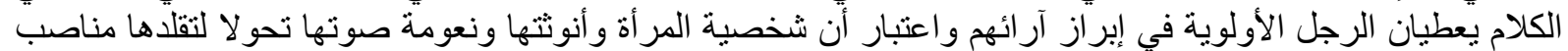

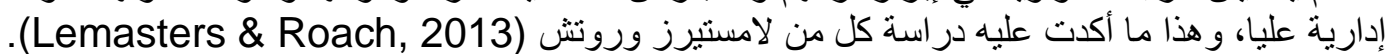

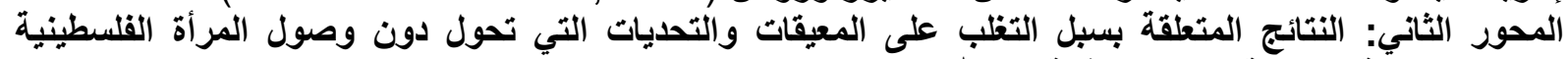

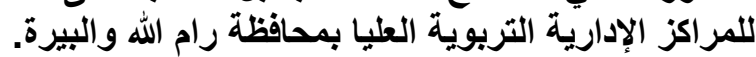

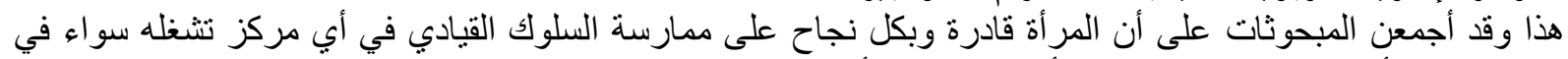

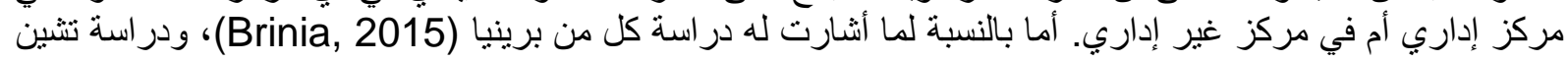

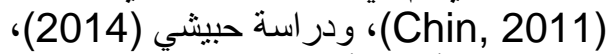
وبعد طرح أحد الأسئلة على المبحوثات المتمثل في " ما الأمور التي يمكن عملها للتغلب على المعوقات المختلفة

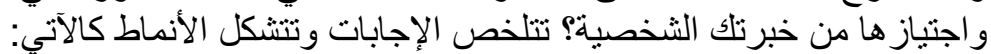

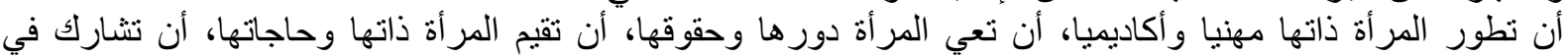

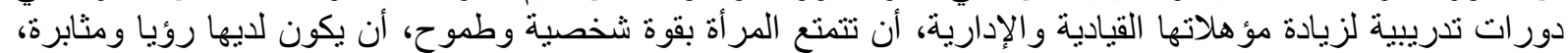

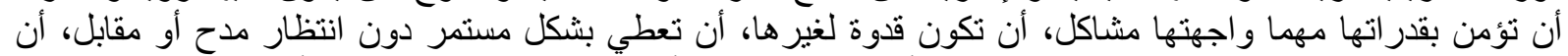

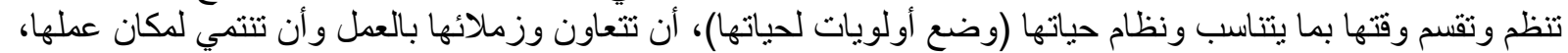

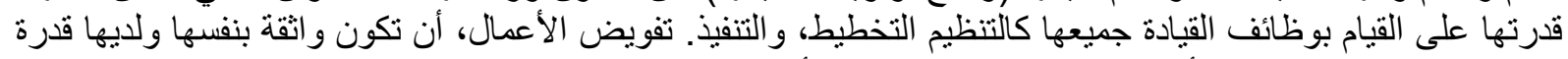

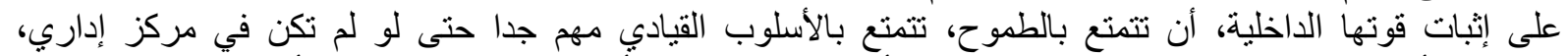

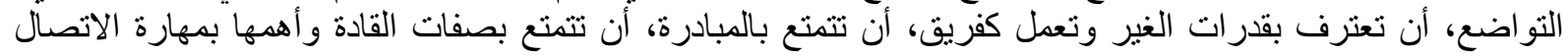




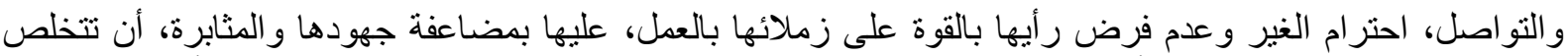

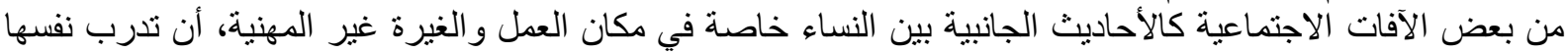

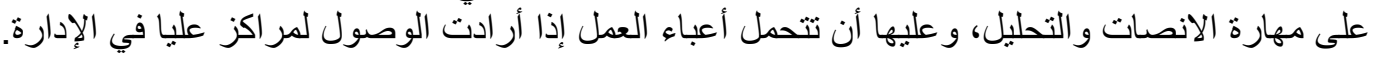

التوصيات

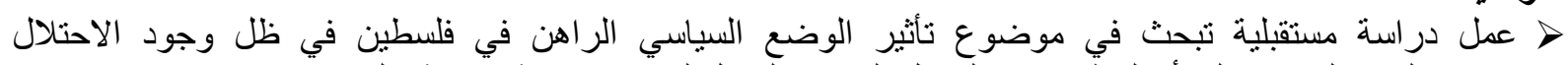

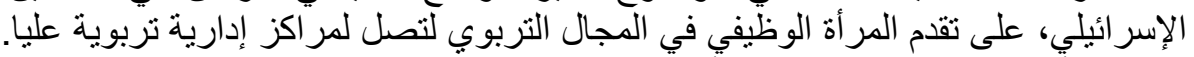

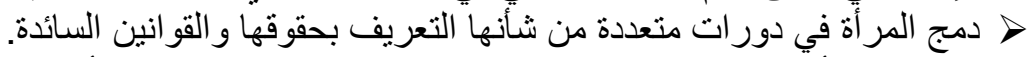

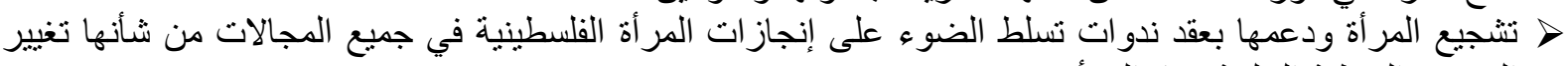

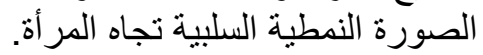

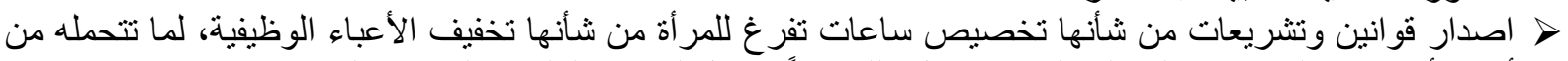

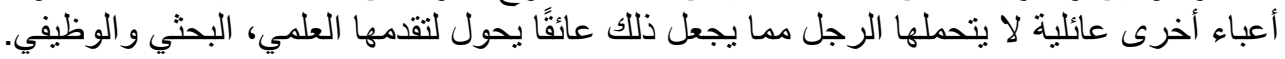

$$
\begin{aligned}
& \text { قائمة المراجع العربية } \\
& \text { البدري، طارق عبد الحميد. (2015). الأساليب القيادية والإدارية في المؤسسات التعليمية (ط.2). عمان، الأردن: دار } \\
& \text { الفكر. } \\
& \text { البشابشة، عبير. (2016). درجة ممارسة المر أة القبادية في وزارة التربية والتعليم الأردنية للمهار ات الإبداعبة في }
\end{aligned}
$$$$
\text { عملها. وزارة التربية و التعليه. 45(3). 2016. 58-59. }
$$

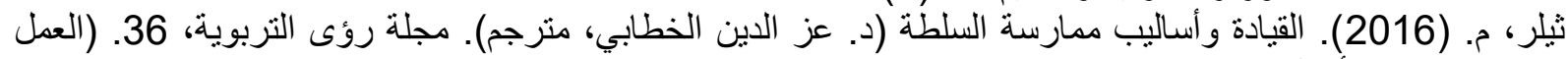
الأصلي نشر سنة 2000).

حبيشي، عادل. (2014). قياس القدرة على القيادة التربوية لمديري ومديرات المدارس الثانوية في محافظة عدن.

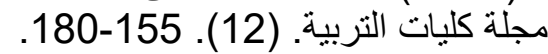

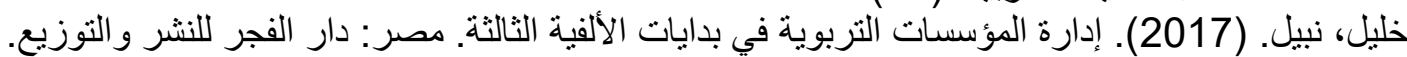

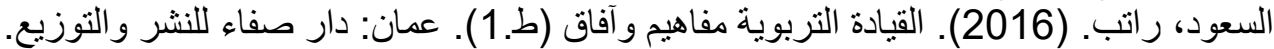

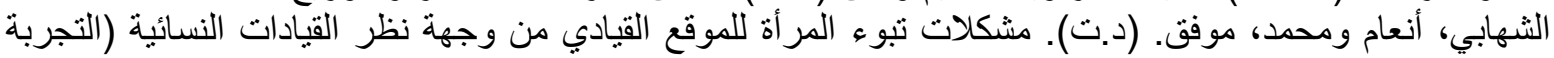

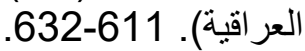

وزارة التربية و التعليم. الدليل الإحصائي السنوي 2019-2020 لمؤسسات التعليم العالي الفلسطيني. رام الله، فلسطين.

قائمة المراجع الأجنبية

Airin, R. (2015). Influencing Factors of Female Representation as School Principals in Indonesia. (Non-Journal). (ERIC Document Reproduction Service No. ED536707.1-14.

Brinia, V. ( 2015). Men VS. Women; Educational Leadership in Primary Schools in Greece: an Empirical Study. International Journal Of Educational Management, 26 (2). 175-191.

Chin, J. (2011). Women and Leadership: Transforming Visions and Current Contexts. The Forum On Public Policy. 1-12.

Flanagan, E. and O'leary, V. (2012). In Encyclopedia of Women and Gender: Sex Similarities and Differences and the Impact of Society on Gender. Oxford, United Kingdom: Elsevier science \& technology. Retrieved from http://search.credoreferences.com/content/entry/estwomen/leadership/0.

Hacifazlioglu, O. (2016). Entry and Transition to Academic Leadership: Experiences of Women Leaders from Turkey and the U.S. Educational Sciences: Theory \& Practice, 10 (4). 2257-2273. 
Kaparou, M. \& Bush, T. (2017). The Career Progress of Women Secondary School Principals in Greece. A Journal Of Comparative Education, 37 (2). 221237. (ERIC Document Reproduction Service No. EJ764039).

Lemasters, L. \& Roach, V. (2012). 3-Dimensional Portrait of the Female CEO. NCPEA, 7(1). Austin State University. 1-12.

Liljia, S. \& Luddeckens, M. (2016). Women in Middle Management in Germany, Sweden and the United Kingdom. Published Master's thesis. Internationalla Handelshogskolan: Hogskolan I Jonkoping.

Rosette, A. \& Toast, L. (2013). Agentic Women and Communal Leadership: How Role Prescriptions Confer Advantage. Journal Of Applied Psychology, 95 (2). 221-235. 